\title{
KVG-Revision: die in der Politik diskutierten Vorschläge
}

\author{
Hanspeter Kuhn, Fürsprecher, stv. Generalsekretär FMH
}

La version française suivra
Die zweite KVG-Revision wurde im Ständerat im Dezember 2001 beraten. Die Vorlage wird zurzeit in der vorberatenden Kommission des Nationalrats behandelt. Sie soll in der Dezembersession 2002 ins Nationalratsplenum kommen.

Im Spitalbereich sollen sich Kassen und Kantone in einem Zwischenschritt die Spitalfinanzierung in einem fixen Schlüssel teilen, wie dies der Bundesrat in der Botschaft vom September 2000 vorgeschlagen hat. Damit sollen wenigstens die fruchtlosen Diskussionen (und ebenso fruchtlosen Bundesratsentscheide) um den Kostendeckungsgrad im Spital beendet werden: Kantone und Kassen haben dann beide ein Interesse, dass die stationäre Behandlung möglichst günstig ist.

Diese Lösung ändert hingegen noch nichts am Schwarzpeter-Spiel zwischen allen Beteiligten, ob der Patient stationär oder ambulant behandelt werden kann und soll. Hierzu will die Nationalratskommission den Bundesrat rascher als der Ständerat verpflichten, einen Vorschlag für eine monistische Spitalfinanzierung zu erarbeiten, also zu zeigen, wie die heutigen kantonalen Spitalsubventionen für die stationäre Behandlung konkret umgelenkt werden können, damit der Patient im Spitalbett für die Kasse nicht mehr künstlich billiger ist als der ambulant behandelte.

Für die ambulante Medizin stehen sich in der KVG-Revision zurzeit im wesentlichen drei Konzepte gegenüber:

\section{Abschaffung des sogenannten «Kontrahierungszwangs»}

Der Begriff ist so schillernd wie irreführend. Das heutige KVG kennt gar keinen Zwang, einen Vertrag abzuschliessen. Die Kassen sind vielmehr direkt durch das Gesetz verpflichtet, die Behandlungskosten der Patientinnen und Patienten zu übernehmen, die von ihrer Ärztin oder ihrem Arzt im Rahmen des KVG zum vertraglich oder staatlich festgesetzten Tarif betreut werden. Für die Abschaffung dieser gesetzlichen Pflicht der Kassen hat sich im Dezember 2001 der Ständerat ausgesprochen, und dies wird im Nationalrat von einem Teil der Bürgerlichen vorgeschlagen. Diese Lösung wird direkt aus den USA der 1980er Jahre importiert - die Kassen sollen im Prinzip frei entscheiden, mit welchen ambulanten Leistungserbringern sie zusammenarbeiten wollen und mit welchen nicht. Die Wettbewerbskommission soll den Wettbewerb garantieren. Im Unterschied zu den USA soll der Kanton die Fiktion einer sozialen Krankenversicherung aufrecht erhalten, indem er dafür sorgt, dass alle Versicherer genügend Leistungserbringer auf ihrer Liste haben, also die «Versorgung sicherstellen». Konsequenterweise könnte und müsste in diesem Marktsystem auf Tarife verzichtet werden: Den TARMED braucht es nicht, an seine Stelle treten marktgerechte Preise. (Die im Parlament diskutierten Vorschläge sind hier allerdings inkonsequent. Ständerat wie bürgerliche Parlamentarier im Nationalrat haben bisher vergessen, die Gesetzesartikel 43-48 KVG betreffend Tarife ersatzlos zu streichen.)

\section{Staatliche Planung für High-Tech-Apparate, Aufhebung des Kontrahierungszwangs für Spezialisten, evtl. Globalbudget, evtl. Einheitskasse, evtl. unbefristete Bedürfnisklausel}

Von linker Seite stammt die Idee, den Kontrahierungszwang gegenüber Spezialisten abzuschaffen und eine Grossgeräteplanung für die ambulante Medizin einzuführen. Weiter liegt der vom Parlament 1991-1994 und in der ersten KVG-Revision abgelehnte Vorschlag eines ambulanten Globalbudgets erneut auf dem Tisch. Die mehrheitlich sozialdemokratischen Gesundheitsdirektorinnen und -direktoren der Romandie schlagen vor, für die Grundversicherung eine Einheitskasse zu gründen. Und jemand aus der CVP schlägt vor, die Bedürfnisklausel für ambulante Medizin dauerhaft einzuführen.

\section{Vernünftige Zusammenarbeit von} Versicherern und Leistungserbringern

Einige sozialliberale Bürgerliche schlagen diese Linie vor, die weder alle Macht den Kassen geben will noch das Heil in reiner staatlicher Planwirtschaft sucht. TARMED soll eine Chance gegeben werden, eine effiziente Medizin sowohl über den Tarif im engeren Sinn wie auch über Qualitätsvereinbarungen und verbesserte Wirtschaftlichkeitsprüfungen zu fördern. 